\title{
Silver Valley, an Innovation Ecosystem Supporting Longevity
}

\author{
By Nicolas Menet
}

F rance is one of the most evolved countries in the world regarding the care of older adults. We enjoy a "network of excellence," also referred to as the Silver Economy, which encourages innovators, prestigious universities, companies, and multinational corporations in France to create groundbreaking innovations to address our needs and create a new economy with a beneficial effect on our competitiveness. In 2016, France also passed specific legislation called Adaptation de la Société au Vieillissement (Adapting Society to Aging), also referred to as the Loi ASV (ASV Law). For the first time, seniors are not the ones who have to adapt to society and try to integrate into it; rather, it is up to society to include them and transform itself to better integrate them. This legal document is built around three pillars: improving the daily lives of older people; improving the working conditions of homecare providers; and preventing the loss of autonomy. Thanks to these measures, as well as businesses' needs, Silver Valley has become, over the course of 10 years, a major player in the aging field in France and in Europe.

Silver Valley is an innovation ecosystem dedicated to supporting longevity in society. It's the only one of its kind in France. Silver Valley's objective is to accelerate the development and launching of innovative solutions designed to improve the well-being of seniors. With more than 300 members, 46 percent of which are startups and 54 percent of which are large international corporations such as Microsoft, Silver Valley has grown exponentially in line with the urgent need to act for older adults, who will soon make up more than 30 percent of Western Europe's overall population.

This cluster and its members strive to better understand the societal changes associated with aging. To do so, in 2018 Silver Valley created the Open Lab, a unique sociological mechanism in France integrating about 10,000 people aged 55 to 92, who share their aging experiences throughout the year and explore with us the associated innovation pockets. This community allows us to test, experiment, and carry out innovation projects, and then collect users' final feedback, which is brought back into the heart of the innovation process. The Open Lab is pursuing the objective of reinforcing the relevance of its products and service offerings. This mechanism has two main components: a user component and a life situation component. Its purpose is to restore the possible aging configurations, at an individual, collective, or even situational level. In most cases, this process starts with the user component, which allows for the correct calibration of needs, such as product and service content, marketing, pricing, or communication. For the Open Lab's innovative candidates, the results are true indicators of their project's acceptability for future clients. The experiment's second phase includes the "life situation" component. Many situations are available: homes, accessible housing, home adaptations, retirement homes, Etablissements d'Hébergement pour Personnes Agées Dépendantes (Housing Establishments for Dependent Elderly Persons), Unités de Soin Longue Durée (Long-Term Care Units), and even palliative care units. Depending on the innovation project, we choose the most adapted location, as well as the most relevant target, to experiment in real situations with the product or service intended for dependent or autonomous older people, caregivers, or professionals. The experimentation process aims to produce a concrete deliverable with recommendations and actual optimizations, which will be used to enhance the product or service, as well as improve credibility with potential funders or partners. As such, the Open Lab has become a real instrument for improvement in the provision of suggested solutions, as well as an enhancement tool for the business it generates. And it is thanks to such human and technical wealth that Silver Valley can contribute to inventing tomorrow's offers: offers that are really adapted to emerging needs and that can, therefore, find their market. We are combining three positive actions into one: meeting people's needs, improving the lives of senior citizens, and finding clients for our entrepreneurs.

In addition, Silver Valley chose to integrate its service offerings into a concrete program called Être Entrepreneur (Being an Entrepreneur) 


\section{"Silver Valley is an innovation ecosystem dedicated to supporting longevity in society. It's the only one of its kind in France."}

to benefit from the know-how and ideal conditions of French innovation. Silver Valley now wishes to make its startups real, innovating, perennial, and consolidated companies with the capacity to be established worldwide and answer the industrial demand from large companies. Concretely, this program, dedicated to entrepreneurship, offers individual and collective assistance aimed at allowing innovators to strengthen their management skills and to be in contact with actors capable of helping them create a highly effective, value-based production system. Coaches, investors, and experienced businessmen, and also lawyers, accountants, backers, and managers, are all some of the professions needed to support innovators' management skills as a prerequisite to the creation of a healthy, viable, and perennial economic fabric. Within this program, innovators focus on the business plan, business model, liquidity plan, and legal and administrative issues, as well as the company director's stance.

Finally, large companies, often international in nature, are in urgent need of innovation, particularly with respect to emerging markets such as that of the elderly. Within this cluster, large groups, as well as public administrations and local communities, come to learn more about the new challenges of aging, discover new innovation markets, and incubate their own projects dedicated to better aging. As a result, all the different economic, social, and political players are reunited with the common goal of supporting each other and creating tomorrow's longevity in society.

Combining three programs, namely Open Lab, Être Entrepreneur, and the Programme Grands Groupes (Large Companies Program), allows innovators focusing on aging, including both startups and large companies, to turn the vision of the aging economy into a concrete reality.

The Silver Economy is no longer in its early stages, and it is essential to act efficiently, while preserving the dignity and respecting the wishes of the elderly, as well as any associated economic interests. When the field was created in 2013, the focus was on research and innovation, whereas now the priority is to find the industry's leaders of tomorrow. Such a priority is all the more urgent considering that Asia is following France's measures with immense interest, as it is looking for ways to meet, over a very short span of time, the needs and expectations of more than 500 million of its pensioners by 2030 .

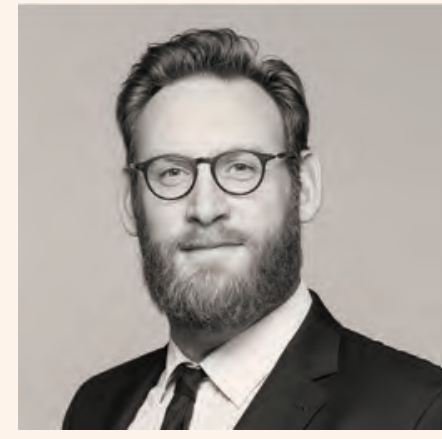

Nicolas Menet GENERAL MANAGER SILVER VALLEY, FRANCE 